\title{
Using fasting plasma glucose concentrations to screen for gestational diabetes mellitus: prospective population based study
}

\author{
Daniele Perucchini, Ursin Fischer, Giatgen A Spinas, Renate Huch, Albert Huch, Roger Lehmann
}

\begin{abstract}
Departments of
Obstetrics and

Gynaecology,

University Hospital

Zurich, CH-8091

Zurich, Switzerland

Daniele Perucchini

senior registrar in

obstetrics

Ursin Fischer

general practitioner

Renate Huch

professor of obstetrics

Albert Huch

professor of obstetrics

Department of

Internal Medicine,

Division of

Endocrinology and

Diabetes, University

Hospital Zurich

Giatgen A Spinas

professor of medicine

Roger Lehmann

senior registrar in

diabetology

Correspondence to:

R Lehmann

Roger.Lehmann@

dim.usz.ch
\end{abstract}

BMJ 1999;319:812-5

\begin{abstract}
Objective To evaluate whether measuring fasting plasma glucose concentration is an easier screening procedure for gestational diabetes mellitus than the 1 hour $50 \mathrm{~g}$ glucose challenge test.

Design Prospective population based study. Setting Outpatient clinic in a university hospital. Participants 520 pregnant women (328 (63\%) white, 99 (19\%) Asian, 31 (6\%) African, 62 (12\%) others) with mean age 28.4 (SD 0.2; range 17-45) years. All underwent a glucose challenge test between the 24th and 28th gestational week, followed by a diagnostic 3 hour $100 \mathrm{~g}$ oral glucose tolerance test within one week. This was done irrespective of the result of the challenge test.

Main outcome measure Receiver operating curves were used to determine the best cut off values for screening with fasting plasma glucose concentrations. Results Fasting plasma glucose concentration at a threshold value of $4.8 \mathrm{mmol} / \mathrm{l}$ and the glucose challenge test with a threshold value of $7.8 \mathrm{mmol} / 1$ yielded sensitivities of $81 \%$ and $59 \%$ respectively and specificities of $76 \%$ and $91 \%$ respectively. Measuring fasting plasma glucose concentration as a screening procedure required a diagnostic test in 30\%,

compared with $14 \%$ when the challenge test was used. Conclusions Measuring fasting plasma glucose concentrations using a cut off value of $\geq 4.8 \mathrm{mmol} / \mathrm{l}$ is an easier screening procedure for gestational diabetes than the $50 \mathrm{~g}$ glucose challenge test and allows $70 \%$ of women to avoid the challenge test.
\end{abstract}

\section{Introduction}

Gestational diabetes mellitus is a common medical complication and metabolic disorder in pregnancy, occurring in $1-14 \%$ of patients depending on the population described and the criteria used for diagnosis. ${ }^{1-4}$ It is associated with an increased incidence of fetal macrosomia, pre-eclampsia, and caesarean section in pregnancy. ${ }^{56}$ Type 2 diabetes develops in $30-50 \%$ of women with gestational diabetes who are followed up long term. ${ }^{78}$ Increasing pregnant women's carbohydrate intolerance is associated with a graded increase in adverse maternal and fetal outcomes. ${ }^{5910}$ This was emphasised in the large blinded trial conducted by the Toronto tri-hospital gestational diabetes project. This trial showed an unequivocal graded relation between the fasting plasma glucose concentration (and other glucose concentrations at different time points during the oral glucose tolerance test) and a wide variety of adverse outcomes. ${ }^{5}$ Identifying women susceptible to gestational diabetes is particularly important not only to prevent perinatal morbidity but also to improve long term outcomes for the mother and her child. ${ }^{11-13}$ We aimed to evaluate whether measuring fasting plasma glucose concentration is an easier screening procedure for gestational diabetes than the $50 \mathrm{~g}$ glucose challenge test in a prospective, population based study, as previously suggested. ${ }^{14}$

\section{Participants and methods}

All pregnant women with a singleton pregnancy in the University Hospital Zurich (772, over a study period of 21 months, 1995-7) were eligible to participate in this prospective study. The only exceptions were women with pre-existing diabetes and those not examined by an obstetrician before the 24th gestational week. Only deliveries after the 28th week of gestation were included in the analysis. The study was approved by the hospital's ethics committee.

\section{Participants' characteristics and risk factors}

Obstetric history (including repeated abortion, gestational diabetes, pre-eclampsia, macrosomic infant(s), and congenital anomalies), family history of diabetes, and weight before pregnancy were obtained from all patients. We assessed potential clinical risk factors-for example, smoking - that could adversely affect maternal and fetal outcome. Body mass index (weight $(\mathrm{kg}) /\left(\right.$ height $\left.(\mathrm{m})^{2}\right)$ ) before pregnancy was calculated by using the most recent self reported weight before conception. Race information was categorised as white (European) $(63 \%$; 328), Asian (19\%; 99), African $(6 \% ; 31)$, and others $(12 \% ; 62)$. The mean age of the 520 women included in the analysis was 28.4 (SD 0.2 ; range $17-45$ ) years, and the mean body mass index was 23.8 (SD 0.2).

\section{Glucose tolerance tests and diagnostic criteria}

The women were given a standard, 1 hour, $50 \mathrm{~g}$ glucose challenge test between the 24th and 28th gestational week, with a venous plasma blood glucose measurement 1 hour later. The women had to record the time of last food intake, although the challenge was performed irrespective of this or of the time of day. Regardless of the results of the challenge, all patients were asked to return for a 3 hour, diagnostic, $100 \mathrm{~g}$ oral glucose tolerance test within the next week. The tolerance test was performed in the morning after a 12 hour overnight fast and 3 days of 150-200 g (minimum) carbohydrate diet. Gestational diabetes was diagnosed if two or more values of the tolerance test equalled or exceeded the thresholds proposed by Carpenter and Coustan and adopted by the fourth international workshop conference on gestational diabetes (table). ${ }^{3}{ }^{15}$ 
Threshold values of most commonly used criteria to diagnose gestational diabetes mellitus ${ }^{23} 15$

\begin{tabular}{lcc}
$\begin{array}{l}\text { Time } \\
\text { points }\end{array}$ & $\begin{array}{c}\text { World Health Organisation* } \\
\text { (75 g glucose) }\end{array}$ & $\begin{array}{c}\text { Fourth international workshop } \\
\text { conference on gestational diabetes }_{\text {mellitus }^{3 *}} \text { (75 g or 100 g glucose) }\end{array}$ \\
\hline Fasting & $\mathrm{NA}$ & $5.3 \mathrm{mmol} / \mathrm{l}$ \\
\hline 1 hour & $\mathrm{NA}$ & $10.0 \mathrm{mmol} / \mathrm{l}$ \\
\hline 2 hour & $7.8 \mathrm{mmol} / \mathrm{l}$ & $8.6 \mathrm{mmol} / \mathrm{l}$ \\
\hline 3 hourt & $\mathrm{NA}$ & $7.8 \mathrm{mmol} / \mathrm{l}$
\end{tabular}

$\mathrm{NA}=$ not applicable.

${ }^{*}$ Venous plasma glucose concentrations determined by glucose oxidase method.

†Not required for the $75 \mathrm{~g}$ glucose load according to the fourth international workshop conference on gestational diabetes mellitus.

\section{Biochemical analysis}

Venous plasma glucose concentration was determined by a hexokinase method (Hitachi 747, Hoffmann LaRoche, Basle, Switzerland).

\section{Statistical analysis}

Data were analysed with STATISTICA FOR wINDOws software (Statsoft, 1997, Tulsa, Oklahoma). Relations among different groups and variables were analysed with the Student's $t$ test and the Mann-Whitney U test where appropriate; $\mathrm{P}<0.05$ was considered significant. Sensitivity, specificity, and predictive values were calculated using a $2 \times 2$ contingency table. Receiver operating curves were used to construct a graphic representation of the relation between sensitivity and specificity of a laboratory test over all possible diagnostic cutoff values. ${ }^{16-18}$

\section{Results}

\section{Participants}

Altogether, 558 (72.3\%) of 772 eligible women gave informed oral consent to participate in the study. After the initial glucose challenge test the women proceeded with the diagnostic oral glucose tolerance test. Thirty eight $(6.8 \%)$ women were excluded because of vomiting after the $100 \mathrm{~g}$ glucose intake $(8(1.4 \%))$, protocol violation (for example, prior food intake, smoking, plasma glucose concentration measured too late; $18(3.2 \%)$ ), or incomplete data (for example, one or several glucose measurements missed, refusal to finish the tolerance test; $12(2.2 \%))$. The demographic characteristics of women who were excluded or not willing to participate in the study were not different from those of the study population.

\section{Prevalence of gestational diabetes}

Gestational diabetes was diagnosed in 53 women $(10.2 \%)$. The condition was slightly more prevalent in Asian (16\% (16/99), $\mathrm{P}<0.05)$ or African women (13\% $(4 / 31), P=0.39)$ than in white women $(8 \%(26 / 328))$. As expected, women with the condition were older (>30 years: $77 \%$ v 46\%; $\mathrm{P}<0.001$ ) and more overweight (body mass index >25.0: $54 \%$ v 32\%; $\mathrm{P}=0.001$ ) than women with normal glucose tolerance.

\section{Fasting plasma glucose concentrations}

A detailed analysis using receiver operating curves showed that the best cut off value for using fasting plasma glucose concentration as a screening test for gestational diabetes was $4.8 \mathrm{mmol} / \mathrm{l}$ (figure). Universal screening using a threshold value for fasting plasma glucose of $4.8 \mathrm{mmol} / \mathrm{l}$ yielded a sensitivity of $81 \%$ and a specificity of $76 \%$. Altogether, $30 \%$ (155) of women had a value $\geq 4.8 \mathrm{mmol} / \mathrm{l}$ and would have had to proceed with the tolerance test. Lowering the threshold value to $4.4 \mathrm{mmol} / \mathrm{l}$ would not have missed any case of gestational diabetes. The sensitivity for the $4.4 \mathrm{mmol} / 1$ threshold was $100 \%$, the specificity $39 \%$. If this threshold value were used, however, $55 \%(285 / 520)$ of all women tested would have been false positives and $65 \%$ $(\mathrm{n}=338)$ would have had to proceed with the tolerance test, compared with $22 \%$ false positive results if a cut off value of $4.8 \mathrm{mmol} / 1$ were used.

\section{$50 \mathrm{~g}$ glucose challenge test}

Receiver operating curves were used for determination of the best cut off values in the glucose challenge test (figure). A cut off value of $7.8 \mathrm{mmol} / 1$ yielded a sensitivity of $59 \%$ and a specificity of $91 \%$. A cut off value of $7.5 \mathrm{mmol} / \mathrm{l}$, as used by several investigators, yielded a sensitivity of $61 \%$ and specificity of $88 \%$. The best cut off value for the $50 \mathrm{~g}$ screening test in our analysis was $7.0 \mathrm{mmol} / 1$ (sensitivity $68 \%$, specificity $82 \%$ (figure)). Thirty eight per cent (199) of the women reported food intake up to 1 hour before the challenge test, 32\% (167) between 1 and 2 hours before, and 27\% (141) > 2 hours before; 13 women did not report the time of last food intake. Analysis of the data for the women with food intake $>2$ hours before the challenge test suggested that the sensitivity of the challenge test can be improved by asking women to fast for 2 hours before (sensitivity $100 \%$, specificity $71 \%$, with a cut off value of $7.0 \mathrm{mmol} / \mathrm{l})$.

\section{Diagnostic $100 \mathrm{~g}$ oral glucose tolerance test}

Forty six $(8.9 \%)$ of all the women had a fasting plasma glucose concentration of $\geq 5.3 \mathrm{mmol} / \mathrm{l}$, of whom 27 $(5.2 \%)$ had been diagnosed with gestational diabetes.

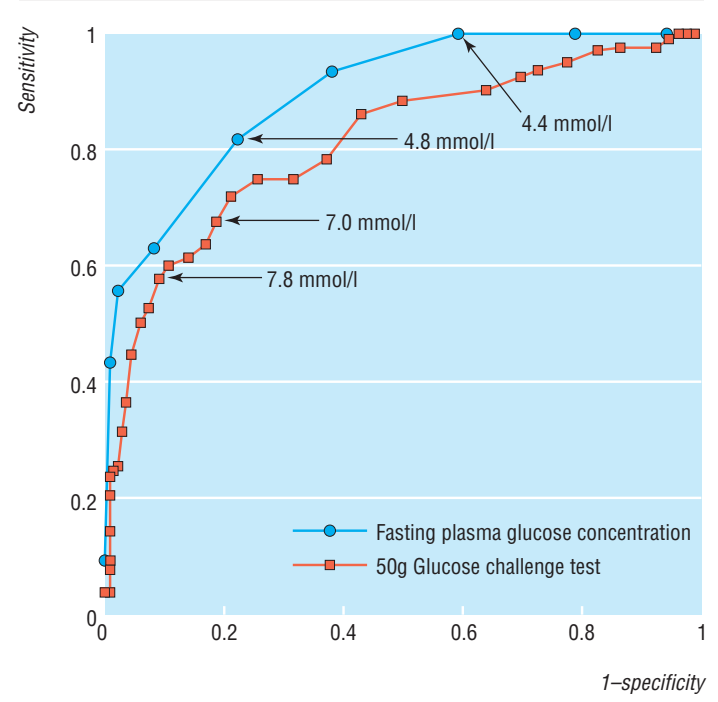

Receiver operating characteristics of two different screening procedures for gestational diabetes (fasting plasma glucose concentration and $50 \mathrm{~g}$ glucose challenge test independent of prior food intake). The area under the curve is 0.897 for fasting plasma glucose concentration and 0.815 for the glucose challenge test. The true positive rates (sensitivity) versus the false positive rates (1-specificity) are plotted for the cut off values (each data point is $0.2 \mathrm{mmol} / \mathrm{l}$ apart) 
Seventy six $(14.6 \%)$ women had a 1 hour value of $\geq 10.0 \mathrm{mmol} / \mathrm{l}$, of whom $50 \quad(9.6 \%$ of all $)$ had gestational diabetes. Fifty two women $(10 \%)$ had a 2 hour value of $\geq 8.6 \mathrm{mmol} / \mathrm{l}$, of whom $39(7.5 \%)$ had gestational diabetes. In 37 patients $(7.1 \%)$ the condition was diagnosed by a raised 1 hour and 2 hour value-that is, $69.8 \%$ of women with gestational diabetes mellitus. The 3 hour value was above the threshold $(\geq 7.8 \mathrm{mmol} / \mathrm{l})$ in only 16 women $(3.1 \%$ of all women), and gestational diabetes was diagnosed in 14 of them $(2.3 \%)$. Four out of $53(7.6 \%)$ cases of gestational diabetes would have been missed by omitting the 3 hour value from analysis, yielding a sensitivity of $92.4 \%$

\section{Discussion}

Although new diagnostic criteria for diabetes mellitus outside pregnancy have been adopted by the American Diabetes Association ${ }^{19}$ and the World Health Organisation, ${ }^{20}$ a consensus is still lacking on both diagnostic and screening criteria for gestational diabetes mellitus. The new criteria for diagnosis of diabetes outside pregnancy rely on fasting plasma glucose concentration ${ }^{19} 20$ or the $75 \mathrm{~g}$ oral glucose tolerance test. ${ }^{20}$

\section{Screening with plasma glucose concentrations}

Using fasting plasma glucose concentration with a threshold value of $4.8 \mathrm{mmol} / 1$ yielded a $22 \%$ better sensitivity than the $50 \mathrm{~g}$ glucose challenge test with a threshold value of $7.8 \mathrm{mmol} / \mathrm{l}$ and an acceptable specificity $(76 \% v 91 \%)$. The low sensitivity of the challenge test in our study might be explained by the fact that a high percentage of women reported food intake up to 2 hours before the test, which is known to reduce the test's sensitivity owing to the Staub-Traugott effect. ${ }^{21}$

Lowering the threshold value to $4.4 \mathrm{mmol} / \mathrm{l}$, a value similar to the $4.1 \mathrm{mmol} / \mathrm{l}$ cut off point for referring women to the tolerance test as proposed by Mortensen et al, ${ }^{22}$ would not have missed any case of gestational diabetes in our study and had a sensitivity of $100 \%$, but it had a low specificity $(39 \%)$ and a very high percentage of false positive results $(55 \%)$. The use of this threshold, therefore, would have required $65 \%$ of women to have a tolerance test. A drawback of the studies of Sacks et $\mathrm{al}^{14}$ and Mortensen et $\mathrm{al}^{22}$ lay in the selection criteria, which might have biased selection of patients towards those at increased risk of gestational diabetes. Currently, only one large prospective trial evaluating the use of fasting plasma glucose concentrations to screen for gestational diabetes has been published. ${ }^{23}$ In that study, however, only $45 \%$ of the women studied were white, with most being of African $(14 \%)$ or mixed ethnicity (41\%), and the body mass index was higher than in our study. As the prevalence of gestational diabetes is influenced by ethnic background, obesity, and physical activity of a population, diagnostic threshold values and screening methods might have to be adjusted to the local community. There are several reasons why using fasting plasma glucose concentration might be more robust and transferable to other populations. Firstly, the concentration is not influenced by gestational age in pregnancies with normal glucose tolerance, in contrast to the 1 or 2 hour plasma glucose after the oral
- Identifying women susceptible to gestational diabetes is particularly important not only to prevent perinatal morbidity but also to improve long term outcomes for the mother and her child

- Measuring fasting plasma glucose concentration is an easier screening procedure for gestational diabetes than the 1 hour $50 \mathrm{~g}$ glucose challenge test

- With a threshold value of $4.8 \mathrm{mmol} / \mathrm{l}$ it yields a sensitivity of $81 \%$ and a specificity of $76 \%$

- Measuring fasting plasma glucose concentration allows $70 \%$ of women to avoid a glucose challenge test

tolerance test. ${ }^{24}$ Secondly, the concentration has a much lower variation and better reproducibility than postprandial values. ${ }^{25}$ Thirdly, the suggested threshold value of $4.8 \mathrm{mmol} / 1$ used in the present study is closely similar to the values for screening suggested by the studies of Sacks et al (4.7-4.9 mmol/l) and Reichelt et al $(4.5-4.7 \mathrm{mmol} / \mathrm{l})^{14}{ }^{23}$-these studies were conducted in populations with a different ethnic background (west coast United States and Brazil respectively).

\section{Two step $v$ one step screening procedure}

The two step screening approach for gestational diabetes has often been criticised as being more cumbersome than the one step test. We propose universal screening for gestational diabetes using a fasting plasma glucose concentration of $\geq 4.8 \mathrm{mmol} / \mathrm{l}$ between the 24th and 28th gestational week as an easier screening procedure. Any woman with a concentration above the threshold can proceed directly to the diagnostic oral glucose tolerance test. In addition, the 3 hour value can be omitted with a relatively small loss of sensitivity, as recently proposed for the 75 g diagnostic oral tolerance test. ${ }^{3}$ Our data confirm the validity of this recommendation also for the $100 \mathrm{~g}$ diagnostic oral tolerance test. Our proposed screening procedure, based on using fasting plasma glucose concentration and, if necessary, the diagnostic test, would be, therefore, a 2 hour, one step procedure, comparable to the 2 hour oral tolerance test as proposed by the World Health Organisation (table). With the fasting plasma glucose as a screening procedure, $30 \%$ of women have to be referred for the oral tolerance test, compared with $14 \%$ of those screened with the $50 \mathrm{~g}$ challenge test with a cut off value of $7.8 \mathrm{mmol} / \mathrm{l}$. The higher rate of oral tolerance tests associated with screening with fasting plasma glucose concentration is more than compensated for by the avoidance of the challenge test in all the patients.

Contributors: RL had the original idea for the study, designed the protocol, performed most of literature searches, discussed core ideas about the study design, interpreted the results, and wrote the paper. DP participated in the design of the protocol, performed literature searches, supervised patient management, discussed core ideas about the study design, and participated in data analysis and interpretation of the results. UF collected and analysed the clinical data and performed the statistical analysis. GAS participated in the design of the study, discussed core ideas of the paper, and edited the paper. RH discussed core ideas of the paper 
and edited the paper. $\mathrm{AH}$ obtained the funding and discussed core ideas of the paper. RL is the guarantor for the paper.

Funding: University Hospital Zurich.

Competing interests: None declared.

1 Dooley SL, Metzger BE, Cho NH. Gestational diabetes mellitus. Influence of race on disease prevalence and perinatal outcome in a US population. Diabetes 1991;40(suppl 2):25-9.

2 World Health Organisation Expert Committee on Diabetes Mellitus Diabetes mellitus:report of a WHO study group. Geneva: WHO, 1985.

3 Metzger BE, Coustan DR. Summary and recommendations of the fourth international workshop-conference on gestational diabetes mellitus. Diabetes Care 1998;21(suppl 2):B161-7.

4 Magee MS, Walden CE, Benedetti TJ, Knopp RH. Influence of diagnostic criteria on the incidence of gestational diabetes and perinatal morbidity. JAMA 1993;269:609-15.

5 Sermer M, Naylor CD, Gare DJ, Kenshole AB, Ritchie JW, Farine D, et al Impact of increasing carbohydrate intolerance on maternal-fetal outcomes in 3637 women without gestational diabetes. The Toronto trihospital gestational diabetes project. Am J Obstet Gynecol 1995;173:14656.

6 Coustan DR, Imarah J. Prophylactic insulin treatment of gestational diabetes reduces the incidence of macrosomia, operative delivery, and birth trauma. Am J Obstet Gynecol 1984;150:836-42.

7 Coustan DR. Gestational diabetes. Diabetes Care 1993;16(suppl 3):8-15.

8 Mestman JH, Anderson GV, Guadalupe V. Follow-up study of 360 subjects with abnormal carbohydrate metabolism during pregnancy. Obstet Gynecol 1972;39:421-5.

9 Tallarigo L, Giampietro O, Penno G, Miccoli R, Gregori G, Navalesi R. Relation of glucose tolerance to complications of pregnancy in nondiabetic women. $N$ Engl J Med 1986;315:989-92.

10 Langer O, Brustman L, Anyaegbunam A, Mazze R. The significance of one abnormal glucose tolerance test value on adverse outcome in pregnancy. Am J Obstet Gynecol 1987;157:758-63.

11 Eriksson UJ. Lifelong consequences of metabolic adaptations in utero? Diabetologia 1996;39:1123-5.

12 Purdy LP, Metzger BE. Influences of the intrauterine metabolic environment on adult disease: what may we infer from size at birth? Diabetologia 1996;39:1126-30.

13 Silverman BL, Metzger BE, Cho NH, Loeb CA. Impaired glucose tolerance in adolescent offspring of diabetic mothers. Relationship to fetal hyperinsulinism. Diabetes Care 1995;18:611-7.
14 Sacks DA, Greenspoon JS, Fotheringham N. Could the fasting plasma glucose assay be used to screen for gestational diabetes? J Reprod Med 1992;37:907-9.

15 Carpenter MW, Coustan DR. Criteria for screening tests for gestational diabetes. Am J Obstet Gynecol 1982;144:768-73.

16 Beck JR, Shultz EK. The use of relative operating characteristic (ROC) curves in test performance evaluation. Arch Pathol Lab Med 1986;110:1320. (Published correction appears in Arch Pathol Lab Med 1986 110:958.)

17 Bortheiry AL, Malerbi DA, Franco LJ. The ROC curve in the evaluation of fasting capillary blood glucose as a screening test for diabetes and IGT. Diabetes Care 1994;17:1269-72.

18 Engelgau MM, Aubert RE, Thompson TJ, Herman WH. Screening for NIDDM in nonpregnant adults. A review of principles, screening tests, and recommendations. Diabetes Care 1995;18:1606-18.

19 Expert Committee on the Diagnosis and Classification of Diabetes Mellitus. Report of the expert committee on the diagnosis and classification of diabetes mellitus. Diabetes Care 1997;20:1183-97.

20 Alberti K, Zimmet PZ. Definition, diagnosis and classification of diabetes mellitus and its complications. Part 1: diagnosis and classification of diabetes mellitus-provisional report of a WHO consultation. Diabet Med 1998;15:539-53.

21 Lewis GF, McNally C, Blackman JD, Polonsky KS, Barron WM. Prior feeding alters the response to the 50 -g glucose challenge test in pregnancy. The Staub-Traugott effect revisited. Diabetes Care 1993;16:1551-6.

22 Mortensen HB, Molsted PL, Kuhl C, Backer P. A screening procedure for diabetes in pregnancy. Diabetes Metab 1985;11:249-53.

23 Reichelt AJ, Spichler ER, Branchtein L, Nucci LB, Franco LJ, Schmidt ML. Fasting plasma glucose is a useful test for the detection of gestational diabetes. Diabetes Care 1998;21:1246-9.

24 Weiss PAM. Der orale Glukosetoleranztest (oGTT) in der Schwangerschaft. Nicht-diabetogene Einflüsse und Methodenvergleich. Gynäkolog 1998;13:12-24.

25 Mooy JM, Grootenhuis PA, de Vries H, Kostense PJ, Popp-Snijders C, Bouter LM, et al. Intra-individual variation of glucose, specific insulin and proinsulin concentrations measured by two oral glucose tolerance tests in a general Caucasian population: the Hoorn study. Diabetologia $1996 ; 39: 298-305$

(Accepted 2 June 1999)

\author{
Abstract \\ Objectives To investigate the association between the \\ duration of exclusive breast feeding and the \\ development of asthma related outcomes in children \\ at age 6 years. \\ Design Prospective cohort study. \\ Setting Western Australia. \\ Subjects 2187 children ascertained through antenatal \\ clinics at the major tertiary obstetric hospital in Perth \\ and followed to age 6 years. \\ Main outcome measures Unconditional logistic \\ regression to model the association between duration \\ of exclusive breast feeding and outcomes related to \\ asthma or atopy at 6 years of age, allowing for several \\ important confounders: sex, gestational age, smoking \\ in the household, and early childcare. \\ Results After adjustment for confounders, the \\ introduction of milk other than breast milk before 4 \\ months of age was a significant risk factor for all \\ asthma and atopy related outcomes in children aged \\ 6 years: asthma diagnosed by a doctor (odds ratio \\ $1.25,95 \%$ confidence interval 1.02 to 1.52 ); wheeze \\ three or more times since 1 year of age $(1.41,1.14$ to \\ $1.76)$; wheeze in the past year $(1.31,1.05$ to 1.64$)$; \\ sleep disturbance due to wheeze within the past year
}

(1.42, 1.07 to 1.89$)$; age when doctor diagnosed asthma (hazard ratio 1.22, 1.03 to 1.43 ); age at first wheeze $(1.36,1.17$ to 1.59$)$; and positive skin prick test reaction to at least one common aeroallergen (1.30, 1.04 to 1.61 )

Conclusion A significant reduction in the risk of childhood asthma at age 6 years occurs if exclusive breast feeding is continued for at least the 4 months after birth. These findings are important for our understanding of the cause of childhood asthma and suggest that public health interventions to optimise breast feeding may help to reduce the community burden of childhood asthma and its associated traits.

\section{Introduction}

Asthma is the leading cause of admission to hospital in Australian children and its prevalence is increasing. ${ }^{2}$ Susceptibility to asthma may be increased by factors present early in life. ${ }^{3}$ These include being male, low birth weight, preterm birth, young maternal age, maternal smoking and, possibly, early cessation of exclusive breast feeding. ${ }^{4}$ Environmental allergens including house dust mite, grasses, or pollens may also cause sensitisation. Conversely, early exposure to respiratory infections may be protective. ${ }^{56}$
TVW Telethon Institute for Child Health Research, PO Box 855, West Perth, Western Australia, Australia 6872

W H Oddy senior research officer P G Holt

National Health and Medical Research Council senior principal research fellow

P D Sly

National Health and Medical Research Council principal research fellow A W Read senior research officer continued over

BMJ 1999;319:815-9 